\title{
Effect of alcohol and tobacco use on vascular dementia: a matched case control study
}

\author{
This article was published in the following Dove Press journal: \\ Vascular Health and Risk Management \\ I4 November 201 I \\ Number of times this article has been viewed
}

\section{Paul Y Takahashi \\ Casey R Caldwell \\ Paul V Targonski}

Primary Care Internal Medicine, Mayo Clinic, Rochester, MN, USA
Correspondence: Paul Y Takahashi Division of Primary Care Internal Medicine, Mayo Clinic, 200 First Street SW, Rochester, MN 55905, USA

Tel + I 50728425 I I

Fax + I 5072660036

Email takahashi.paul@mayo.edu
Background: Vascular dementia is the second most common type of dementia in the United States. The underlying association of tobacco and alcohol with vascular dementia is not completely understood.

Purpose: Determine the relationship of tobacco and alcohol use with the development of vascular dementia $(\mathrm{VaD})$.

Methods: This was a matched case-control study of subjects living in Olmsted County, $\mathrm{MN}$. Cases of VaD were identified through medical record abstraction using conventionally accepted definitions of $\mathrm{VaD}$, using the National Institute of Neurological Disorders and Stroke and the Association Internationale pour la Recherche et l'Ensignement en Neurosicences (NINDS-AIRENS) criteria and were matched to controls by gender and age within 3 years among persons free of dementia on the index date. Exposure data for alcohol and tobacco use were abstracted by trained nurses, along with demographic, lifestyle, cerebrovascular, cardiovascular, and vascular comorbid disease characteristics. Matched conditional logistic regression for univariate and multivariate evaluation of the association of tobacco and alcohol use with VaD was utilized.

Results: Current alcohol exposure was associated with a decreased risk of VaD with an odds ratio of 0.48 (95\% confidence interval: $0.31-0.74)$. This protective effect of alcohol was seen in men, women, and subjects under 80 years of age. Tobacco use was not associated with VaD in univariate and multivariate analysis, and stratified analysis did not reveal any subgroup-specific associations between tobacco use and $\mathrm{VaD}$ in the study population.

Conclusion: Current alcohol use appears to have protective effects against the development of vascular dementia. The effects are more pronounced in subjects under age 80 . This may reflect the direct vascular effects of alcohol on the vascular system or may represent a surrogate for better social or functional status. Previous alcohol use was not protective. Tobacco use was not a risk factor for $\mathrm{VaD}$ status, which was possibly an indication of survivorship bias in the cohort.

Keywords: vascular dementia, elderly, alcohol, tobacco

\section{Introduction}

Vascular dementia ( $\mathrm{VaD})$ is a common and increasingly recognized illness that has some identifiable lifestyle comorbid risk factors. VaD involves cognitive decline with underlying pathological lesions of vascular ischemia or occlusion. ${ }^{1}$ In a Midwestern autopsy study, VaD represented the second most common form of dementia, following Alzheimer's disease, and may account for up to $25 \%$ of dementia in the United States. ${ }^{2}$ Internationally, there are differences in VaD prevalence. The Japanese suffer a higher prevalence of $\mathrm{VaD}$ than Americans, ${ }^{3}$ perhaps demonstrating a cultural or ethnic component to the illness. The importance of lifestyle issues in these cultural 
differences remains incompletely characterized. Many of the lifestyle risk factors for vascular dementia have treatable components or may be protective. Thus, there is a need to understand how lifestyle issues, like tobacco and alcohol use, influence the development of vascular dementia.

Despite partial understanding of some of the risk factors for stroke, many questions remain about potential risk factors for $\mathrm{VaD}$. One cannot assume that the risk factors for stroke and $\mathrm{VaD}$ are the same. The objective of this study was to determine the differences in risk from lifestyle exposures between individuals with $\mathrm{VaD}$ and controls without $\mathrm{VaD}$. We evaluated the exposure to tobacco and alcohol in both $\mathrm{VaD}$ cases and in the control group. We hypothesized that tobacco use was a risk factor, as it is a risk factor for other vascular illnesses. ${ }^{4} \mathrm{We}$ further hypothesized that alcohol was protective against dementia. ${ }^{5}$ To answer these questions, we undertook a matched case-control study in patients with welldocumented VaD compared to age-matched controls.

\section{Method}

\section{Study design}

This study utilizes a retrospective, matched case-control analysis of patients living within Olmsted County, MN. The Mayo Clinic medical record system has over 70 years of medical records data from over 6 million patients and has been widely used for epidemiological studies. ${ }^{6}$

\section{Setting}

The Mayo Clinic is the largest health care provider in Southeastern, MN, providing care at the primary, secondary, and tertiary levels. The time ranges included cases and controls from inpatient and outpatient medical care records from 1994-2002. Exposures were included for all times prior to determination of case or control status. All visits to Mayo Clinic providers are captured via physician notes, electronic medical records, and through clinical billing in an electronically integrated medical record system. These records are comprehensive and follow the patient throughout their life. Paper and electronic information sources were available and complete for all patients seen during the time of study initiation.

\section{Study subjects}

Population

The US Census estimated Olmsted County's population to be 124,277 in $2000 .^{7}$ Educationally, $38.9 \%$ had a bachelor's degree or higher compared to $27.4 \%$ in the US at large. Of the Olmsted County population, $90.3 \%$ were white, $4.3 \%$ Asian and 2.7\% black or African American. ${ }^{7}$

\section{Cases}

Cases were defined as patients with a clinically documented diagnosis of vascular dementia. Records were screened for any one of 40 diagnoses suggestive of dementia. The incident cases of $\mathrm{VaD}$ were drawn from these records. The diagnostic information to make the determination was obtained from medical histories, neuroimaging studies, and clinical diagnoses. The diagnosis of dementia was based on the National Institute of Neurological Disorders and Stroke and the Association Internationale pour la Recherche et 1'Ensignement en Neurosicences (NINDS-AIREN) criteria for $\mathrm{VaD}^{8}{ }^{8}$ The date of diagnosis of $\mathrm{VaD}$ for the case represented the index date for each case-control pair. If a control developed dementia during the time frame of the study, after having been selected as a control, he or she was included as a case with a uniquely matched control. Status as a previous control was retained relative to that individual's matched case.

\section{Controls}

Controls were dementia-free in the medical records with the corresponding case index data and were matched 1:1 for age ( \pm 2 years), gender, and Mayo Clinic registration number. Matching for registration number allowed for similar access times to the medical system.

\section{Exclusion criteria}

Individuals with any diagnosis of dementia before 1994 were excluded from the study. Patients who refused authorization for medical records review were also excluded.

\section{Exposure variables}

The lifestyle exposure variables were tobacco and alcohol use. We used a dichotomous representation of ever (yes/no), previous (yes/no), and current (yes/no) for tobacco use. The specific types of tobacco use were as described: cigarette use, cigar use, pipe, and chew/smokeless tobacco use. The subjects also reported the number of years of tobacco use. Alcohol use had a dichotomous representation of ever (yes/no), previous (yes/no), and current (yes/no). The subjects self-reported the number of years of alcohol use. The amounts of alcohol or tobacco were also recorded. Specific types of alcohol included beer, wine, and hard liquor. All lifestyle information was based on self-report from the patient. Patient personal history obtained from the prior date closest to the index date was used.

The characteristics of the cohort were also collected. Comorbid medical illnesses were defined based upon clinical 
diagnosis or by guidelines. Cerebrovascular disease was defined as medically diagnosed history of stroke and/or transient ischemic attacks (TIA). Stroke or TIA was defined as ever or never, prior to the index date. Myocardial infarction was evaluated clinically and was defined as ever or never, prior to the index date. Congestive heart failure, angina, and atrial fibrillation were defined as ever or never, prior to the index date. Hypertension was defined based upon JNC VII criteria of systolic blood pressure (SBP) greater than $140,{ }^{9}$ prior to the index date. The exposure used was the presence or absence of hypertension. Diabetes was diagnosed clinically and was defined as ever or never, prior to the index date. Educational years were determined based upon the last year of education.

\section{Data acquisition}

The primary information abstracted included medical diagnoses and surgical information, which was coded using the International Classification of Disease adapted code for hospitals (H-ICDA). Alcohol and smoking information were collected using a self-reported questionnaire that patients completed prior to a medical evaluation. A trained nurse abstractor obtained and recorded the information on an electronic spreadsheet (Excel, v 2003; Microsoft Corp, Redmond, WA). The abstractor was blinded to the study hypothesis and was not involved with any analysis of data. Further information on the case and control exposures was obtained from the Mayo Clinic records-linkage system.

We attempted to reduce bias by blinding the data abstractors to the purpose of the study. The subjects reported alcohol and smoking use in a clinical setting; thus, there was potential for under-reporting its use. Recall bias would be minimized because the questionnaires asked about recent, as well as previous use. Despite these efforts, the effects of bias were likely present.

\section{Sample size}

All available subjects who met the criteria within the time frame described were enrolled within the case-control study, because of the relatively uncommon nature of the case status (VaD). Two hundred and five matched pairs were included within the analysis.

\section{Data analysis}

Simple descriptive statistics were calculated for demographic and exposure data among cases and controls, as well as for gender strata. The primary method of comparison between cases and controls utilized the odds ratio as a surrogate for relative risk with $95 \%$ confidence intervals $(95 \% \mathrm{CI})$. We utilized conditional logistic regression for analysis of the exposure variables between cases and controls. Subjects with missing information were excluded from analysis. We performed stratified conditional logistic regression for both gender and for age $(<80$ years and 80 years and above). All analyses were performed on Epi Info (v 3.5; Centers for Disease Control, Atlanta, GA).

\section{Results}

In the study, 1736 potential subjects were identified using the initial evaluation from their medical records. From this group, the investigators identified 205 cases of VaD using standard criteria as noted above. The records of 1531 patients did not meet the criteria after medical record review. The schematic distribution is shown in Figure 1. The 205 pairs included 121 pairs of female subjects and 84 pairs of male subjects with an overall mean age of 81.9 years (SD: 7.79 years). The average length of educational involvement was 12.3 years (SD: 3.25 years). Comparing males and females, males had a lower age of 78.2 years (SD: 7.88 years) compared to 84.1 years (SD: 6.86 years) in females. The average educational achievement did not differ between the genders. The most common comorbid health condition was hypertension in the cohort, with $72.9 \%$ of cases and controls having hypertension. The full demographic and descriptive information of the overall cohort is noted in Table 1. Lifestyle exposures are listed in Table 2. These tables indicate the missing data. Missing data levels were high for specific alcohol type, and for the remainder of the variables between 8 and 17 were missing. The absolute numbers of exposures in cases and controls are listed.

Tobacco exposure (current, previous, ever) was not different between cases and controls in the univariate analysis

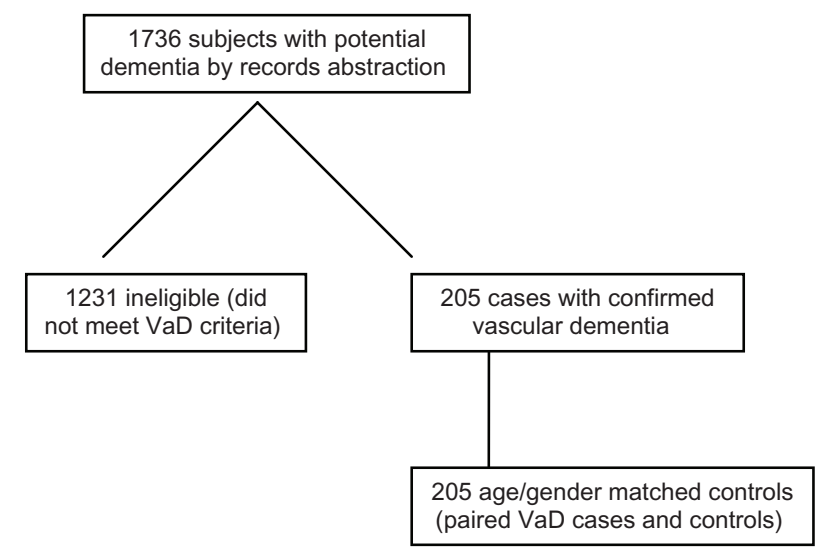

Figure I Flow of subjects till final determination of cohort. 
Table I Demographic overview of cases of vascular dementia and controls in 205 matched pairs

\begin{tabular}{|c|c|c|c|}
\hline & $\begin{array}{l}\text { Overall } \\
(n=410)\end{array}$ & $\begin{array}{l}\text { VaD cases } \\
(n=205)\end{array}$ & $\begin{array}{l}\text { Controls } \\
(n=205)\end{array}$ \\
\hline \multicolumn{4}{|l|}{ Demographics } \\
\hline Male (\%) & $168(4 \mid .0)$ & $84(41.0)$ & $84(41.0)$ \\
\hline Age in years (SD) & $81.9(7.79)$ & $82.1(7.73)$ & $81.6(7.85)$ \\
\hline Education in years $(S D)$ & $12.3(3.25)$ & $12.0(3.35)$ & $12.6(3.13)$ \\
\hline $\begin{array}{l}\text { Body mass index, } \\
\mathrm{kg} / \mathrm{m}^{2}(\mathrm{SD})\end{array}$ & $26.2(5.02)$ & $25.8(4.50)$ & $26.5(5.40)$ \\
\hline Obese, yes/no (\%) & $175(53.4)$ & $76(5 \mid .4)$ & $99(55.0)$ \\
\hline \multicolumn{4}{|c|}{ History of comorbid health conditions } \\
\hline Stroke $(\%)$ & $148(37.0)$ & $133(65.8)$ & $15(7.6)$ \\
\hline TIA (\%) & $80(20.0)$ & $66(32.7)$ & $14(7.1)$ \\
\hline $\mathrm{CHF}(\%)$ & $88(21.8)$ & $60(29.3)$ & $28(14.1)$ \\
\hline Angina (\%) & $130(32.2)$ & $70(34.1)$ & $60(30.3)$ \\
\hline Atrial fibrillation (\%) & $88(21.8)$ & $58(28.3)$ & $30(15.1)$ \\
\hline Previous MI (\%) & $78(19.3)$ & $4 I(20.0)$ & $37(18.6)$ \\
\hline Hypertension (\%) & 291 (72.9) & 161 (80.1) & $130(65.7)$ \\
\hline Diabetes (\%) & I0I (24.7) & $64(3 \mid .2)$ & $37(18.1)$ \\
\hline
\end{tabular}

Abbreviations: $\mathrm{VaD}$, vascular dementia; $\mathrm{TIA}$, transient ischemic attack; $\mathrm{CHF}$, congestive heart failure; MI, myocardial infarction.

in the overall cohort. There also was no difference between different types of tobacco and VaD status. Lastly, there was no association between years of tobacco use and case or control status. These findings are noted in Table 3. We stratified the cohort by gender (male and female), and we did not find differences in tobacco exposure between cases and controls. Lastly, we stratified the analysis by age (under and over 80 years of age) and again did not find a difference in tobacco use (current, previous, ever). Tobacco frequency or amount of tobacco use also was not significantly different between $\mathrm{VaD}$ cases and controls.

Current alcohol use exposure was protective with an odds ratio of 0.48 (95\% CI: 0.31-0.74). Previous alcohol use and ever alcohol use did not have a significant effect

Table 2 Alcohol and tobacco exposure in a cohort of 410 subjects

\begin{tabular}{llll}
\hline Exposure & Cases & Controls & Missing data \\
\hline Alcohol use & & & \\
Alcohol, current & $53 / 199$ & $86 / 198$ & 13 \\
Alcohol, previous & $149 / 199$ & $153 / 194$ & 17 \\
Alcohol, ever & $149 / 199$ & $154 / 195$ & 16 \\
Beer & $56 / 167$ & $59 / 166$ & 77 \\
Wine & $48 / 167$ & $54 / 166$ & 77 \\
Hard liquor & $75 / 168$ & $94 / 166$ & 76 \\
Tobacco use & & & \\
Tobacco, current & $17 / 198$ & $11 / 199$ & 13 \\
Tobacco, previous & $112 / 201$ & $111 / 201$ & 8 \\
Tobacco, ever & $112 / 201$ & $111 / 201$ & 8 \\
Cigar & $7 / 201$ & $8 / 199$ & 10 \\
Pipe & $12 / 201$ & $9 / 199$ & 10 \\
Chew/snuff & $4 / 201$ & $3 / 199$ & 10 \\
\hline
\end{tabular}

in the overall cohort. Specific alcohol type did not provide protection or serve as a risk in the overall cohort (Table 3). In the gender subgroup analysis, there was a similar protective effect of current alcohol use in both females, with an odds ratio of 0.45 (95\% CI: $0.25-0.84)$, and males, with an odds ratio of 0.52 (95\% CI: $0.28-0.96)$. The remaining types of alcohol use (previous or ever) were not significant in the gender subgroup analysis. The only additional significant value was a slight protective effect of wine use in women with an odds ratio of 0.52 (95\% CI: $0.27-0.99)$. In the age subgroup analysis, current alcohol use did not achieve significance in adults under 80 years of age. It was significant in adults over 80 with an odds ratio of $0.31(95 \%$ CI: 0.15-0.66). All remaining factors for alcohol use were not significantly different between the overall cohort and the age stratified cohort.

The duration of smoking or alcohol use did not show a difference between $\mathrm{VaD}$ cases and controls. The number of years of tobacco use was not associated with VaD status and was similar in both cases and controls. The number of years of alcohol use was also not associated with $\mathrm{VaD}$ between cases and controls. These findings were also found in the subgroup analysis by gender for both alcohol and tobacco use. Stratification by age did not provide additional information on different types of alcohol use or tobacco use.

\section{Discussion}

Vascular dementia remains a growing public health issue as our society faces the demographic tsunami of an aging populace. In the US, the old ( $>65$ years) and the oldest old ( $>80$ years), will continue to expand into 2040, and stand as the fastest growing sections of the population. ${ }^{10}$ The diagnosis of $\mathrm{VaD}$ remains somewhat challenging; however, the NINDS-AIREN criteria currently stand as the accepted standard for VaD diagnosis. Agreement among different providers using NINDS-AIREN criteria has been moderate to good. ${ }^{11}$ In numerous longitudinal studies, increasing age has a strong association with increased prevalence of $\mathrm{VaD} .{ }^{12,13}$ In our study, we found that tobacco use did not contribute to an increased risk of $\mathrm{VaD}$, while current alcohol use was protective.

\section{Tobacco use}

We evaluated tobacco use by looking at current, previous, and ever use, while also looking for a dose effect. All of these risk variables were non-significant, with current use of tobacco having the highest but nonsignificant odds ratio of 1.67 (95\% CI: 0.73-3.81). We evaluated tobacco use by 
Table 3 Univariate analysis of demographic and lifestyle risk factors for 205 matched pairs of vascular dementia cases and healthy controls

\begin{tabular}{|c|c|c|c|c|}
\hline & Odds ratio & Lower $95 \% \mathrm{Cl}$ & Upper $95 \% \mathrm{Cl}$ & $P$ value \\
\hline \multicolumn{5}{|l|}{ Demographics } \\
\hline Age & 1.39 & 1.13 & 1.69 & $0.002^{*}$ \\
\hline Education in years (SD) & 1.00 & 1.00 & 1.001 & 0.87 \\
\hline \multicolumn{5}{|l|}{ Lifestyle alcohol use } \\
\hline Alcohol, current & 0.48 & 0.31 & 0.74 & $0.00 I^{*}$ \\
\hline Alcohol, previous & 0.80 & 0.49 & 1.31 & 0.38 \\
\hline Alcohol, ever & 0.80 & 0.49 & 1.31 & 0.38 \\
\hline Beer, ever & 0.88 & 0.50 & 1.55 & 0.67 \\
\hline Wine, ever & 0.78 & 0.48 & 1.24 & 0.29 \\
\hline Hard liquor, ever & 0.64 & 0.40 & 1.04 & 0.07 \\
\hline Number of years drinking & 0.99 & 0.98 & 1.01 & 0.31 \\
\hline \multicolumn{5}{|l|}{ Tobacco use } \\
\hline Tobacco, current & 1.67 & 0.73 & 3.81 & 0.23 \\
\hline Tobacco, previous & 1.00 & 0.66 & 1.52 & 1.00 \\
\hline Tobacco, ever & 1.00 & 0.66 & 1.52 & 1.00 \\
\hline Cigar, ever & 0.88 & 0.32 & 2.41 & 0.80 \\
\hline Pipe, ever & 1.33 & 0.56 & 3.16 & 0.51 \\
\hline Chew/snuff, ever & 1.00 & 0.20 & 4.95 & 1.00 \\
\hline Number of years smoking & 1.01 & 1.00 & 1.02 & 0.12 \\
\hline
\end{tabular}

Note: *Reached statistical significance.

tobacco type and did not find an association between specific tobacco type and VaD status. Subgroup analysis did not show any significant association between tobacco use and $\mathrm{VaD}$. However, there were some interesting trends that were noted. In the younger and female populations, there was no association between tobacco status and case status. Men who had previously or ever used tobacco seemed to have some trends toward higher odds ratios, but these trends did not reach significance. The older population $(80+$ years old $)$ also seemed to have a trend toward a higher odds ratio of $\mathrm{VaD}$. Our study population represents a primarily white population which differs from other studies. The novel findings in this study include a lack of associations with different age or gender subgroups and with different tobacco products. The lack of a consistent relationship between $\mathrm{VaD}$ and tobacco use may reflect survivorship bias in that patients who smoke and survived to develop $\mathrm{VaD}$ are different to those who died. The apparent protective effects of smoking in Alzheimer's disease and Parkinson disease have been attributed to survivorship bias. ${ }^{14}$

The results of previous studies on the effect of smoking and $\mathrm{VaD}$ have been mixed. Smoking stands as an important risk factor for stroke ${ }^{15}$ and may be a potential risk factor for VaD. However, the direct evidence that smoking affects $\mathrm{VaD}$ has been unimpressive. A recent meta-analysis of the relationship between $\mathrm{VaD}$ and smoking had a nonsignificant odds ratio of 1.35 (95\% CI: $0.90-2.02) .{ }^{16}$ In a Chinese study of six communities, there was an association of $\mathrm{VaD}$ with smoking with a relative risk of 1.98 (95\% CI: $1.53-3.12) .{ }^{17}$ In the Honolulu aging study, there was no significant relationship between current, former, and never smokers and the development of $\mathrm{VaD} .{ }^{18}$ Thus, there is still debate on this issue, with our study showing no relationship between tobacco use and $\mathrm{VaD}$.

\section{Alcohol use}

In our study, current alcohol use was a protective factor with an odds ratio of 0.48 (95\% CI: $0.31-0.74)$. Current alcohol use was protective in the overall cohort as well as within the subgroup analysis of females and in those patients $<80$ years of age. The protective effect seemed strongest in cases younger than 80 years, with an odds ratio of 0.31 (95\% CI: $0.15-0.66)$. Within the overall cohort, there did not appear to be a significant association with either alcohol type or previous alcohol use and VaD. In the subgroups, women seemed to have a protective effect with wine use; however, the remaining subgroups did not have significant values. It appears that current alcohol use, as opposed to previous use, seems to be protective. This could reflect a direct cardiovascular effect of alcohol upon vascular risks. Mild-to-moderate alcohol intake is associated with improved endothelial function. ${ }^{19}$ It could also represent better social support in those individuals who drink socially with other individuals. ${ }^{20}$ The individuals who drink alcohol also possess a higher functional status than those who do not drink. ${ }^{21}$ However, in previous studies, even after adjustment for social economic status and functional 
status, those individuals who have a moderate intake of alcohol have increased longevity. ${ }^{21}$ In our study, if a patient suffers $\mathrm{VaD}$, it is quite possible that they do not have the means or methods of obtaining alcohol for current use.

Alcohol use has had a mixed picture with vascular disease and with memory problems. Alcohol has a neurotoxic effect upon the central nervous system and in excess, can cause alcohol-related dementia. ${ }^{22}$ In a pooled meta-analysis, light-to-moderate alcohol use had a small protective effect for the development of any type of dementia with a relative risk of 0.75 (95\% CI: 0.57-0.98). Heavy alcohol use was not protective and was a risk in this meta-analysis. ${ }^{23} \mathrm{VaD}$ as a specific illness was not evaluated in this meta-analysis. In a large Chinese cohort study, mild-to-moderate alcohol consumption had a larger protective effect for VaD risk with a hazard ratio of 0.31 (95\% CI: 0.19-0.51) compared to Alzheimer's disease with a hazard ratio of 0.63 (95\% CI: $0.55-0.72$ ). Heavy alcohol use was not protective. ${ }^{24}$ In the Canadian Study of Health and Aging, there was no association between weekly alcohol use (beer, wine, or spirits) and the development of $\mathrm{VaD}$. The odds ratios varied between alcohol type; however, the lowest odds ratio was for beer, at 0.61 (95\% CI: 0.31-1.26) and was not significant. ${ }^{25}$ Thus, the relationship with alcohol use has not shown a consistently protective effect in preventing $\mathrm{VaD}$; however, in aggregate, the studies do trend toward a protective effect.

\section{Study limitations}

There are some limitations with the study. In this matched case-control study, one has the advantages of robust medical records with defined criteria. The agreement between electronic abstraction and nurse abstraction tends to favor nurse abstraction, which was performed in this study. ${ }^{26}$ There exists the possibility of missing information which can be an inherent challenge with case-control studies. For the primary outcomes of tobacco or alcohol use, the range of missing information was low at $8-17(2 \%-4 \%)$ missing records per exposure variable. The type of tobacco or alcohol type had more missing records. However, the records were fairly complete and the subjects filled out questionnaires yearly. The biggest challenge involves the self-report of lifestyle issues such as alcohol use. ${ }^{27}$ There may have been a tendency to under-report alcohol or tobacco use to the provider. This could mitigate any real effects or differences between $\mathrm{VaD}$ cases and the controls. In case-control studies, the issue of recall bias is also an important one. The lifestyle questionnaires are asked yearly; thus, the risk of recall bias is reduced.
The generalizability of this study to other populations remains an important question. The population of the study was a largely English-speaking white population. The ability to generalize this to other populations and other countries might be problematic. The weight of the evidence that alcohol is protective in vascular disease is growing. However, there are potentially economic, social, and cultural issues that may influence the type and amount of alcohol taken. Thus, the findings of this study are encouraging, but one must be cautious if the local population differs greatly from the one studied.

\section{Conclusions}

Vascular dementia remains a common and daunting problem that affects many adults in developed and developing countries. This matched case-control study found that current alcohol use may actually reflect a potential protective effect of alcohol on the vascular system. Tobacco use (current, previous, ever), along with type of tobacco use, was not associated with VaD status. This lack of association may be related to a survivorship bias. There clearly remains further work to be performed to fully understand all of the risks for vascular dementia in the older population.

\section{Acknowledgments}

The following individuals helped with collection of the data and information: Sarah Shonyo for data collection and Jody Clikeman for her editorial assistance. The project was primarily supported by grant RO1 HL 73103 from the National Heart, Lung, and Blood Institute.

\section{Disclosure}

The authors report no conflicts of interest in this work.

\section{References}

1. Hachinski V, Iadecola C, Petersen RC, et al. National Institute of Neurological Disorders and Stroke-Canadian Stroke Network vascular cognitive impairment harmonization standards. Stroke. 2006;37(9):2220-2241.

2. Knopman DS, Parisi JE, Boeve BF, et al. Vascular dementia in a population-based autopsy study. Arch Neurol. 2003;60(4):569-575.

3. Ikejima C, Yasuno F, Mizukami K, Sasaki M, Tanimukai S, Asada T. Prevalence and causes of early-onset dementia in Japan: a populationbased study. Stroke. 2009;40(8):2709-2714.

4. Olin JW, Sealove BA. Peripheral artery disease: current insight into the disease and its diagnosis and management. Mayo Clin Proc. 2010; 85(7):678-692.

5. Sinforiani E, Zucchella C, Pasotti C, Casoni F, Bini P, Costa A. The effects of alcohol on cognition in the elderly: from protection to neurodegeneration. Funct Neurol. 2011;26(2):103-106.

6. Melton LJ 3rd. History of the Rochester Epidemiology Project. Mayo Clin Proc. 1996;71(3):266-274.

7. Bureau C. Projected Population of the United States, by Age and Sex: 2000 to 2050. Available from: http://www.census.gov/population/www/ projections/usinterimproj/. Accessed September 1, 2009. 
8. Roman GC, Tatemichi TK, Erkinjuntti T, et al. Vascular dementia: diagnostic criteria for research studies. Report of the NINDS-AIREN International Workshop. Neurology. 1993;43(2):250-260.

9. Chobanian AV, Bakris GL, Black HR, et al. The Seventh Report of the Joint National Committee on Prevention, Detection, Evaluation, and Treatment of High Blood Pressure: the JNC 7 report. JAMA. 2003;289(19):2560-2572.

10. Kinsella K, Wan H. An Aging World: 2008. Washington, DC: US Government Printing Office; 2009. Available from: http://www.census gov/prod/2009pubs/p95-09-1.pdf. Accessed October 25, 2011.

11. Lopez OL, Larumbe MR, Becker JT, et al. Reliability of NINDS-AIREN clinical criteria for the diagnosis of vascular dementia. Neurology. 1994;44(7):1240-1245.

12. Yoshitake T, Kiyohara Y, Kato I, et al. Incidence and risk factors of vascular dementia and Alzheimer's disease in a defined elderly Japanese population: the Hisayama Study. Neurology. 1995;45(6): 1161-1168.

13. Moroney JT, Tang MX, Berglund L, et al. Low-density lipoprotein cholesterol and the risk of dementia with stroke. JAMA. 1999;282(3): 254-260.

14. Riggs JE. The "protective" influence of cigarette smoking on Alzheimer's and Parkinson's diseases. Quagmire or opportunity for neuroepidemiology? Neurol Clin. 1996;14(2):353-358.

15. Shinton R, Beevers G. Meta-analysis of relation between cigarette smoking and stroke. BMJ. 1989;298(6676):789-794.

16. Beckett NS, Peters R, Fletcher AE, et al. Treatment of hypertension in patients 80 years of age or older. $N$ Engl J Med. 2008;358(18): 1887-1898.

17. Juan D, Zhou DH, Li J, Wang JY, Gao C, Chen M. A 2-year follow-up study of cigarette smoking and risk of dementia. Eur J Neurol. 2004;11(4):277-282.
18. Tyas SL, White LR, Petrovitch H, et al. Mid-life smoking and late-life dementia: the Honolulu-Asia Aging Study. Neurobiol Aging. 2003; 24(4):589-596.

19. Suzuki K, Elkind MS, Boden-Albala B, et al. Moderate alcohol consumption is associated with better endothelial function: a cross sectional study. BMC Cardiovasc Disord. 2009;9:8.

20. Ikehara S, Iso H, Yamagishi K, Yamamoto S, Inoue M, Tsugane S. Alcohol consumption, social support, and risk of stroke and coronary heart disease among Japanese men: the JPHC Study. Alcohol Clin Exp Res. 2009;33(6):1025-1032.

21. Lee SJ, Sudore RL, Williams BA, Lindquist K, Chen HL, Covinsky KE. Functional limitations, socioeconomic status, and all-cause mortality in moderate alcohol drinkers. J Am Geriatr Soc. 2009;57(6):955-962.

22. Oslin D, Atkinson RM, Smith DM, Hendrie H. Alcohol related dementia: proposed clinical criteria. Int J Geriatr Psychiatry. 1998;13(4): 203-212.

23. Anstey KJ, Mack HA, Cherbuin N. Alcohol consumption as a risk factor for dementia and cognitive decline: meta-analysis of prospective studies. Am J Geriatr Psychiatry. 2009;17(7):542-555.

24. Deng J, Zhou DH, Li J, Wang YJ, Gao C, Chen M. A 2-year follow-up study of alcohol consumption and risk of dementia. Clin Neurol Neurosurg. 2006;108(4):378-383.

25. Hebert R, Lindsay J, Verreault R, Rockwood K, Hill G, Dubois MF. Vascular dementia: incidence and risk factors in the Canadian study of health and aging. Stroke. 2000;31(7):1487-1493.

26. St Sauver JL, Hagen PT, Cha SS, et al. Agreement between patient reports of cardiovascular disease and patient medical records. Mayo Clin Proc. 2005;80(2):203-210.

27. Del Boca FK, Noll JA. Truth or consequences: the validity of selfreport data in health services research on addictions. Addiction. 2000; 95 Suppl 3:S347-S360.
Vascular Health and Risk Management

\section{Publish your work in this journal}

Vascular Health and Risk Management is an international, peerreviewed journal of therapeutics and risk management, focusing on concise rapid reporting of clinical studies on the processes involved in the maintenance of vascular health; the monitoring, prevention and treatment of vascular disease and its sequelae; and the involvement of

\section{Dovepress}

metabolic disorders, particularly diabetes. This journal is indexed on PubMed Central and MedLine. The manuscript management system is completely online and includes a very quick and fair peer-review system, which is all easy to use. Visit http://www.dovepress.com/ testimonials.php to read real quotes from published authors 